\title{
Bone mineral density gains with a second 12-month course of romosozumab therapy following placebo or denosumab
}

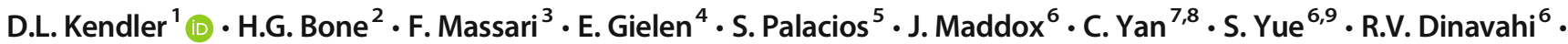 \\ C. Libanati ${ }^{10} \cdot$ A. Grauer ${ }^{6,11}$
}

Received: 19 February 2019 / Accepted: 23 August 2019/Published online: 18 October 2019

(C) The Author(s) 2019

\begin{abstract}
Summary Romosozumab is a therapy that stimulates bone formation and reduces bone resorption. In this study of postmenopausal women with low BMD, a second course of romosozumab following a period off treatment or on denosumab increased or maintained BMD, respectively, and was well tolerated, providing insight into treatment sequence options.

Introduction In patients with high fracture risk, therapies that stimulate bone formation provide rapid BMD gains; currently available agents, parathyroid hormone receptor agonists, are limited to a 2-year lifetime exposure and generally used for a single treatment course. However, for long-term osteoporosis management, a second treatment course may be appropriate. Romosozumab, a therapy with the dual effect of increasing bone formation and decreasing bone resorption, reduces fracture risk within 12 months. Here, we report efficacy and safety of a second romosozumab course.

Methods In this phase 2, dose-finding study, postmenopausal women with low bone mass (T-score $\leq-2.0$ and $\geq-3.5$ ) received romosozumab or placebo (month 0-24) followed by placebo or denosumab (month 24-36); participants then received a year of romosozumab (month 36-48).

Results Of 167 participants who entered the month 36-48 period, 35 had been initially randomized to romosozumab $210 \mathrm{mg}$ monthly. In participants who received romosozumab $210 \mathrm{mg}$ monthly followed by placebo, a second romosozumab course $(n=$ $19)$ increased BMD by amounts similar to their initial treatment (month $0-12)$ at the lumbar spine $(12.4 \% ; 12.0 \%$, respectively) and total hip $(6.0 \%$; $5.5 \%$, respectively). Following denosumab, a second romosozumab course $(n=16)$ increased BMD at the lumbar spine $(2.3 \%)$ and maintained BMD at the total hip. Safety profiles were similar between first and second romosozumab courses.

Conclusions After 12 months off-treatment, a second romosozumab course again led to rapid and large BMD gains. Following denosumab, BMD gains with romosozumab were smaller than with initial treatment. No new safety findings were observed during the second course.
\end{abstract}

Keywords Antiresorptive $\cdot$ Bone-forming agent $\cdot$ Clinical trial $\cdot$ Denosumab $\cdot$ Osteoporosis $\cdot$ Romosozumab

Electronic supplementary material The online version of this article (https://doi.org/10.1007/s00198-019-05146-9) contains supplementary material, which is available to authorized users.

D.L. Kendler

davidkendler@gmail.com

1 Department of Medicine, University of British Columbia, 150-943 West Broadway, Vancouver, BC V5Z 4E1, Canada

2 Michigan Bone and Mineral Clinic, Detroit, MI, USA

3 Instituto de Investigaciones Metabólicas, Buenos Aires, Argentina

4 UZ Leuven, Leuven, Belgium
5 Instituto Palacios, Madrid, Spain

Amgen Inc., Thousand Oaks, CA, USA

Amgen Ltd., Cambridge, UK

8 Present address: Cambridge Statistics Ltd, Cambridge, UK

9 Present address: Atara Biotherapeutics, Westlake Village, CA, USA

10 UCB Pharma, Brussels, Belgium

11 Present address: Corcept Therapeutics, Menlo Park, CA, USA 


\section{Introduction}

Osteoporosis is a chronic condition that requires long-term treatment. Increasing evidence supports treatment strategies designed to improve bone mineral density (BMD) until desired goals have been achieved and maintained in order to reduce the risk of fracture $[1,2]$. Therapies that stimulate bone formation can quickly increase BMD and improve bone microstructure. Such therapies increase bone strength [3], resulting in a rapid reduction in fracture risk $[4,5]$.

In the lifetime management of a patient with osteoporosis, more than one course of a therapy that stimulates bone formation may be clinically warranted. Currently available agents that stimulate bone formation, such as the parathyroid hormone (PTH) analogue teriparatide and the PTH receptor agonist abaloparatide, are limited to a combined 2 years of treatment in a patient's lifetime due to lack of longer-term clinical data and carcinogenicity observed in animal models [6,7], thus generally limiting treatment to a single course of PTH agonist therapy.

Romosozumab is a monoclonal antibody that binds to and inhibits sclerostin. This therapy for osteoporosis has a dual effect of increasing bone formation and decreasing bone resorption $[8$, 9]. The first 12 months of this dose-finding, phase 2 study in postmenopausal women with low bone mass (ClinicalTrials. gov, NCT00896532) evaluated the efficacy and safety of different romosozumab doses $(70 \mathrm{mg}, 140 \mathrm{mg}$, and $210 \mathrm{mg}$ ) administered by subcutaneous (SC) injection at 1- or 3-month intervals to identify the optimal romosozumab regimen [8]. The $210 \mathrm{mg}$ dose of romosozumab administered monthly (QM) for 12 months produced greater increases in BMD [8], and was subsequently selected for further clinical development. This dose regimen was shown to reduce the risk of new vertebral, clinical, nonvertebral, and hip fractures in phase 3 pivotal fracture trials with 12 months of treatment $[4,5]$, including in an alendronate-controlled active-comparator study [5]. In an extension of the phase 2 study, we investigated the effects of switching from romosozumab to placebo or denosumab from month 24 to month 36 and observed that participants receiving denosumab continued to accrue BMD, whereas BMD returned toward pretreatment levels when romosozumab was switched to placebo without follow-on therapy [10]. At month 36 of this phase 2 study, all participants were switched from placebo or denosumab to a second course of romosozumab $210 \mathrm{mg}$ QM for another 12 months. Here, we report the efficacy and safety results of this second course of romosozumab treatment.

\section{Methods}

\section{Study design}

This phase 2, international, multicenter, randomized, placebocontrolled study enrolled postmenopausal women aged 55-
85 years with a low BMD (T-score of $\leq-2.0$ and $\geq-3.5$ at the lumbar spine, total hip, or femoral neck) [8]. Key exclusion criteria were history of vertebral fracture or fragility fracture of the wrist, humerus, hip, or pelvis after 50 years of age; history of metabolic bone disease; and a serum level of 25hydroxyvitamin $\mathrm{D}<20 \mathrm{ng} / \mathrm{mL}$. The design and primary results for this study have been previously published [8].

Treatment groups in the romosozumab double-blind period (month 0 to 24 ), the denosumab extension period (month 24 to 36), and the romosozumab second-course period (month 36 to 48 ) are presented in Fig. 1. In brief, participants were first randomly assigned $(1: 1: 1: 1: 1: 1: 1: 1)$ to double-blind treatment with placebo or one of five SC regimens of romosozumab $(70 \mathrm{mg}$ or $140 \mathrm{mg}$ or $210 \mathrm{mg}$ $\mathrm{QM}$, or $140 \mathrm{mg}$ or $210 \mathrm{mg}$ every 3 months [Q3M]), openlabel oral alendronate $70 \mathrm{mg}$ weekly (QW), or SC teriparatide $20 \mu \mathrm{g}$ daily for 12 months [8]. Thereafter, participants in the romosozumab and placebo groups continued their assigned treatment for an additional 12 months. At month 24, participants entered a 12month extension period and were rerandomized $(1: 1)$ within their treatment groups to double-blind treatment with SC denosumab $60 \mathrm{mg}$ or placebo every 6 months (Q6M). Women in the alendronate group switched to SC romosozumab $140 \mathrm{mg}$ QM at month 12, were randomized in the denosumab extension period, and completed the study at month 36 . Women in the teriparatide group ended study participation at month 12 . Women who were initially randomized to placebo or romosozumab and completed the extension at month 36 were eligible to enter a 12 month second-course period with SC romosozumab $210 \mathrm{mg}$ QM through month 48. As participants in the alendronate and teriparatide groups did not participate in the second-course period, they were not included in the present analysis. Throughout the study, all women were instructed to take calcium $(\geq 1 \mathrm{~g})$ and vitamin $\mathrm{D}(\geq$ 800 IU) daily. Additional details of the study design have been previously published $[8,10]$.

\section{Study procedures and outcomes}

BMD was measured at the lumbar spine and proximal femur by dual X-ray absorptiometry (Lunar, GE Medical Systems, Madison, WI, USA or Hologic, Hologic Inc., Bedford, MA, USA) at baseline (month 0 ) and months 3, 6, 12, 18, 24, 30, 36, 39, 42, and 48. BioClinica (previously known as Synarc; Newark, CA, USA) analyzed the scans blinded to treatment assignments and provided quality control of the individual scans and densitometers.

Blood was collected and analyzed for serum chemistry, hematology, bone turnover markers, and levels of antiromosozumab antibodies. Levels of the bone formation marker procollagen type $1 \mathrm{~N}$-terminal propeptide (P1NP; 


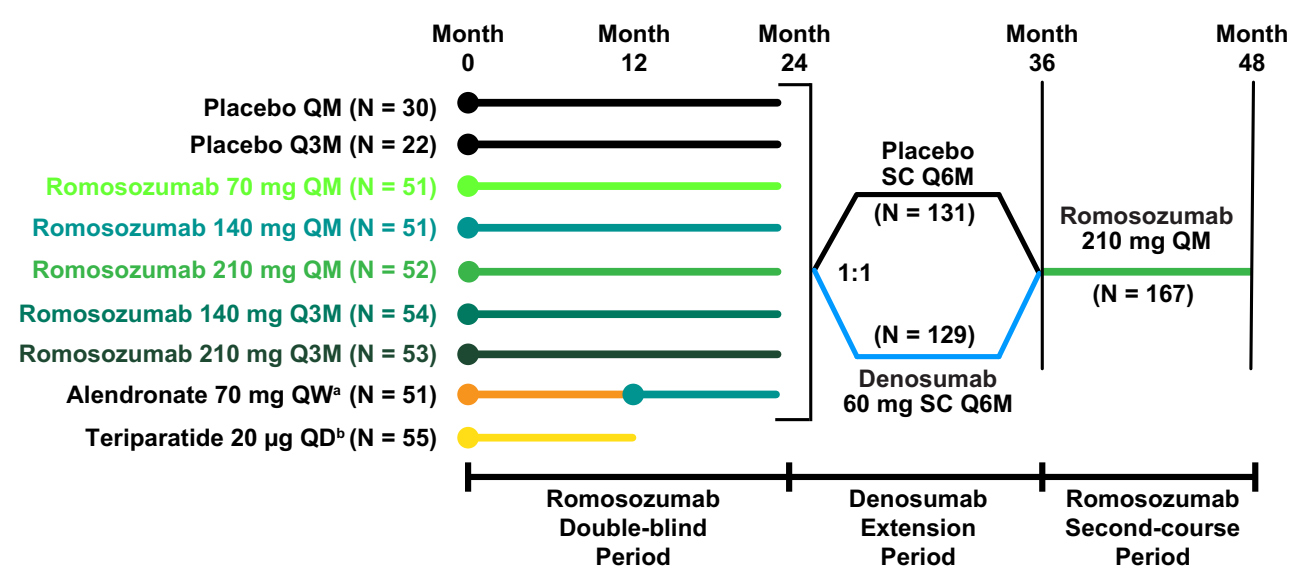

Fig. 1 Study schema. Participants were randomized 1:1:1:1:1:1:1:1 for the first 24 months of treatment. Administration of placebo and the various romosozumab doses was blinded; alendronate and teriparatide were administered open-label. At month 24, participants were rerandomized (1:1) within treatment groups to receive blinded placebo or denosumab $60 \mathrm{mg} \mathrm{SC}$ Q6M for 12 months, followed by a 12month second-course period with romosozumab $210 \mathrm{mg}$ QM. All participants were instructed to take calcium $(\geq 1 \mathrm{~g})$ and vitamin $\mathrm{D}(\geq$

UniQ P1NP RIA, Orion Diagnostica Oy, Espoo, Finland) and the bone resorption marker $\beta$-isomer of $\mathrm{C}$-terminal telopeptide of type 1 collagen $(\beta$-CTX; Serum CrossLaps ELISA, Nordic Bioscience Diagnostics, A/S, Herlev, Denmark) were assessed as previously described [11], through month 36 and then at months 37, 39, 42, 45, and 48 .

Adverse events were reported by individual trial sites. Serum levels of antiromosozumab binding antibodies were assessed at month 0 , month 1 , then every 3 months through month 27 , followed by months $36,39,42,45$, and 48; positive samples were additionally tested for romosozumab neutralizing activity in vitro, as previously described [8].

Results of the study periods up to month 36 have been previously published $[8,10]$. This report focuses on results from the romosozumab second-course period of the study (month 36 to 48 ) with endpoints of the percentage change from month 0 in BMD at the lumbar spine, total hip, and femoral neck, and bone turnover markers (P1NP and $\beta$ CTX), as well as safety analyses of a second course of romosozumab following 12 months of denosumab or placebo together with calcium and vitamin D.

\section{Statistical analysis}

The efficacy analysis set included participants who were initially randomized to SC romosozumab $210 \mathrm{mg}$ QM from month 0 to 24 and enrolled in the romosozumab secondcourse period. We report efficacy of the romosozumab $210 \mathrm{mg}$ QM dose only as this is the dose evaluated in the phase 3 trials [4, 5]; combined results for all romosozumab doses had shown a similar efficacy trend to that of romosozumab $210 \mathrm{mg}$, as previously published [10]. The
800 IU) daily. ${ }^{a}$ Participants randomized to alendronate switched to romosozumab $140 \mathrm{mg} \mathrm{QM}$ at month 12 , were randomized to the denosumab extension period, and completed the study at month 36 ; these participants are not included in the present analysis (month 36 to

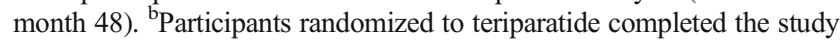
at month 12 and were not included in the present analysis (month 36 to month 48). QD, every day; QM, monthly; Q3M, every 3 months; Q6M, every 6 months; QW, weekly; SC, subcutaneous

safety analysis set included participants initially randomized to either placebo or any dose of romosozumab, who received at least one dose of romosozumab in the second-course period. To assess safety, we compared participants who had their first 12-month exposure to romosozumab during month 36 to 48 (i.e., participants randomized to the two placebo dose groups in month 0 to 24 , followed by placebo or denosumab; $n=27$ ), with participants pooled from all romosozumab groups (month 0 to 24 ) and who had then received romosozumab again in the second-course period (month 36 to $48 ; n=140$ ).

Analysis of the percentage change from month 0 in BMD and bone turnover markers included all participants who had a value at month 0 and at least one measurement on study, with no imputation for missing values. Percentage changes in BMD are presented as means and $95 \%$ confidence intervals. Percentage changes in P1NP and $\beta$-CTX are presented as medians and interquartile ranges. Adverse events were coded using the Medical Dictionary for Regulatory Activities (version 15.1).

\section{Results}

\section{Participant disposition and baseline demographics}

Of the 313 participants enrolled in the romosozumab $(N=$ $261)$ or placebo $(N=52)$ arms during the initial double-blind period of the study, 167 participants entered the romosozumab second-course period (Figs. 1 and 2). Of these, $155(93 \%)$ completed the second-course period (Fig. 2). Of the $11(7 \%)$ participants who discontinued the study during the secondcourse period, the most common reasons were consent 
withdrawn (5 [3\%] participants) and adverse event (4 [2\%] participants). One participant had not undertaken the 48month visit at the time of the analysis; therefore, the analysis included this patient's data for all the time points except data for the 48-month visit. Of the 52 participants initially randomized to romosozumab $210 \mathrm{mg}$ QM, 35 were enrolled in the second-course period (19 had received romosozumab $210 \mathrm{mg}$ QM followed by placebo and 16 had received romosozumab $210 \mathrm{mg}$ QM followed by denosumab) and received a second course of romosozumab (Online Resource Fig. 1). One participant discontinued from each of the two groups. Efficacy results for the 35 participants initially randomized to romosozumab $210 \mathrm{mg}$ QM are reported here since this dose has been evaluated in phase 3 studies $[4,5]$. Safety results are reported for all the 167 participants enrolled in the month 36 to 48 romosozumab second-course period.

Baseline demographics and clinical characteristics were similar between the groups at month 0 (Table 1). At month 36, BMD and bone turnover markers were reflective of the assigned treatment group in the prior 12 months (placebo or denosumab; Table 1).

\section{BMD and bone turnover markers in participants who received romosozumab $210 \mathrm{mg} Q M$ from month 0 to 24}

In participants who switched from romosozumab to placebo in year 3 (month 24 to 36), BMD had declined substantially, albeit not to pretreatment levels [10]. The second course of romosozumab (month 36 to 48 ) resulted in BMD increases at the lumbar spine, total hip, and femoral neck, which were overall similar to the initial BMD increases observed in year 1 of treatment (Fig. 3a, b; Table 2). BMD increased by $12.4 \%$ from month 36 to month 48 (Fig. 3a; Table 2; 12.0\% from month 0 to month 12) at the lumbar spine. At the total hip, BMD increased by $6.0 \%$ from month 36 to month 48 (Fig. 3b; Table 2; 5.5\% from month 0 to month 12). At the femoral neck, BMD increased by $6.3 \%$ from month 36 to month 48 (Table 2; 5.4\% from month 0 to month 12). For the bone formation marker (P1NP) and bone resorption marker $(\beta$ CTX), the percentage changes were similar in the initial romosozumab double-blind and second-course periods in participants who received a second course of romosozumab following placebo (Fig. 3c, d). In these participants, P1NP levels increased in the month following the initiation of the second course of romosozumab (month 37), returned to month 0 levels by month 42 , and thereafter remained below month 0 values through month 48 (Fig. $3 \mathrm{c}$ ). $\beta$-CTX levels decreased toward initial month 0 values by month 39 and fell below month 0 values at months 45 and 48 (Fig. 3d).

In participants who received denosumab from month 24 to month 36, BMD also increased with a second course of romosozumab (month 36 to 48 ) at the lumbar spine, counteracting the expected BMD loss known to occur following denosumab discontinuation (Fig. 3a; Table 2). BMD increased by $2.3 \%$ from month 36 to month 48 (Fig. 3a; Table 2). At the hip measurement sites, BMD was maintained (Fig. 3b; Table 2). At the total hip, the increase from the original study baseline was $7.3 \%$ from month 0 to month 36 , then was unchanged from month 36 to month 48 (Fig. 3b; Table 2). At the femoral neck, the increase from the original baseline was $6.7 \%$ from month 0 to month 36 and remained unchanged from month 36 to month 48 (Table 2). P1NP levels, which had been reduced during denosumab treatment, gradually increased during the second course of romosozumab, reaching month 0 values by month 42 and increasing beyond the original baseline by month 48 (Fig. $3 c)$. $\beta$-CTX levels, which were suppressed during denosumab treatment, increased from month 36 , reaching month 0 values by month 39 to further increase above baseline until month 42 and then return toward baseline by month 48 (Fig. 3d).

\section{Safety for all participants enrolled in the month $\mathbf{3 6}$ to 48 romosozumab second-course period}

The adverse event profile in the second course of romosozumab $210 \mathrm{mg}$ QM (month 36 to 48 ) was similar to the first course of romosozumab $210 \mathrm{mg}$ QM (month 0 to 12; Table 3) [8]. For the 140 participants who received a second course of romosozumab from month 36 to month 48 after being treated with a first course of romosozumab (any dose and schedule) for the first 24 months, the subject incidence of adverse events was $84.3 \%$ (118/140 participants) in the second-course period and $80.0 \%$ (28/35 participants) for the subset of participants who had received a first course of romosozumab $210 \mathrm{mg} \mathrm{QM}$ dose during month 0 to month $24(n=35$; Table 3). The incidence of adverse events in participants who had received a first course of romosozumab followed by placebo and then received a second course of romosozumab was generally comparable to that of participants who had received a first course of romosozumab followed by denosumab and then received a second course of romosozumab: $83.3 \%$ (60/72 participants) and 85.3\% (58/68 participants), respectively. The incidence of adverse events was $88.9 \%$ (24/27 participants) in participants who received a first course of romosozumab during month 36 to month 48 (Table 3).

Serious adverse events were reported in 7 (5.0\%) participants receiving a second course of romosozumab (breast cancer in 2 participants, lung cancer in 2 participants, myocardial infarction in 1 participant, inguinal hernia in 1 participant, and osteoarthritis in 1 participant) and in 1 (3.7\%) participant receiving her first course of romosozumab in the second-course period (thyroid cancer) (Table 3); none were considered to be treatment related. No fatal events were reported in either group. Serious cardiovascular adverse events in participants 


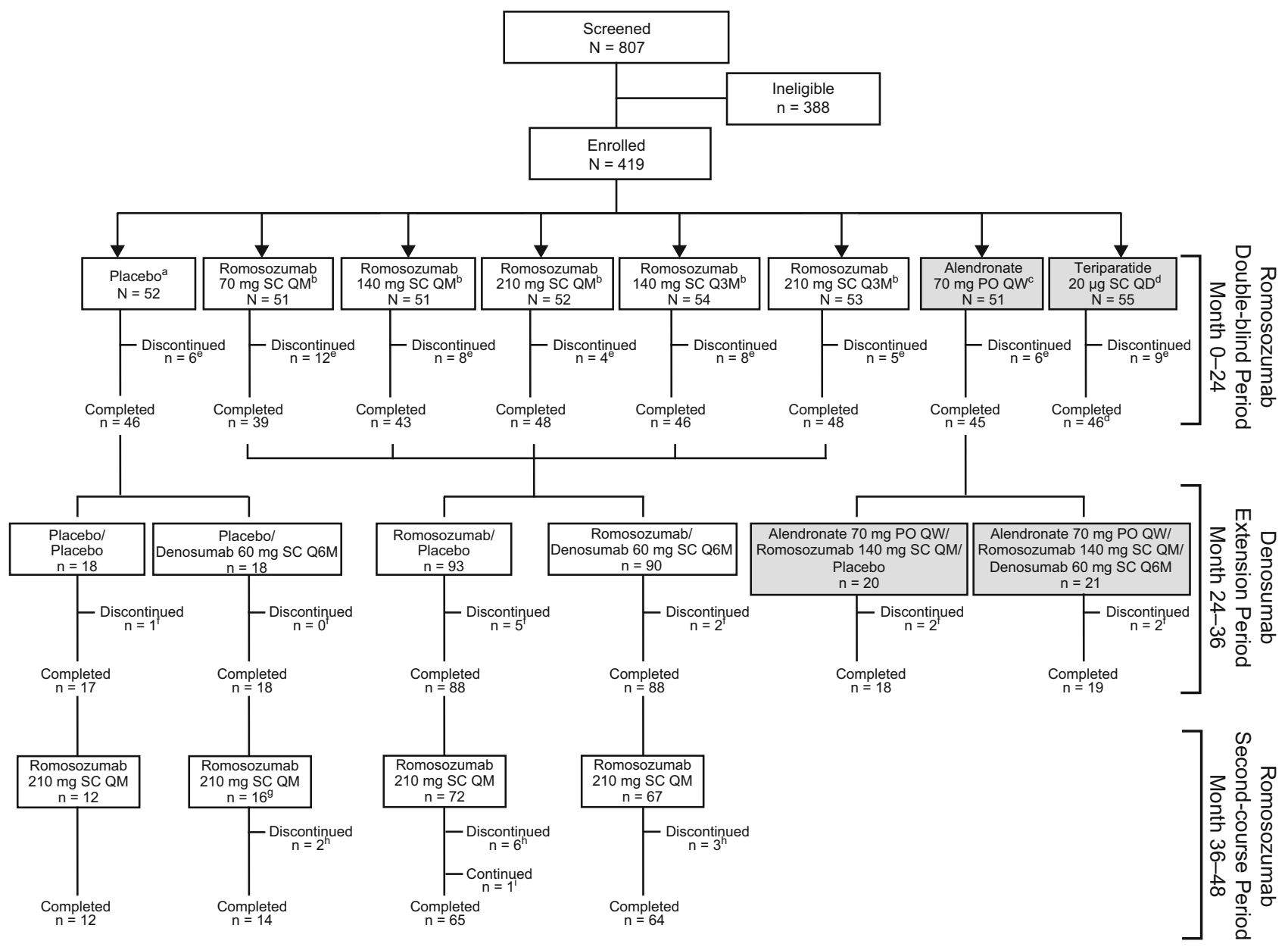

Fig. 2 Disposition for all participants randomized in the study. Participants were randomized 1:1:1:1:1:1:1:1 for the first 24 months of treatment. Administration of placebo and the various romosozumab doses was blinded; alendronate and teriparatide were administered open-label. At month 24, participants were rerandomized $(1: 1)$ within treatment groups to receive blinded placebo or denosumab $60 \mathrm{mg} \mathrm{SC} \mathrm{Q6M} \mathrm{for}$ 12 months, followed by a 12-month second-course period with romosozumab $210 \mathrm{mg}$ QM. Of the participants initially randomized to romosozumab $210 \mathrm{mg}$ QM, 19 placebo-treated and 16 denosumabtreated participants entered the second-course period. All participants were instructed to take calcium ( $\geq 1 \mathrm{~g}$ ) and vitamin $\mathrm{D}$ ( $\geq 800 \mathrm{IU})$ daily. ${ }^{a}$ At month 12, participants initially randomized to receive placebo continued to receive placebo up to month 24 . ${ }^{\mathrm{b}}$ At month 12 , participants initially randomized to receive a specific dose and schedule of romosozumab continued to receive their assigned treatment up to

receiving a second course of romosozumab were low and similar in frequency to those in participants receiving romosozumab during the first course, and also similar in frequency to those in participants receiving placebo from month 0 to month 24 (data not shown).

Adverse events of interest reported during the romosozumab second-course period included hypersensitivity, injection-site reactions, malignancy, and osteoarthritis. Adverse events potentially associated with hypersensitivity were reported in $11(7.9 \%)$ participants receiving a second course of romosozumab and in $2(7.4 \%)$ participants receiving month $24 .{ }^{c}$ At month 12 , participants initially randomized to receive alendronate (gray box) were switched to romosozumab $140 \mathrm{mg}$ QM for 12 months, up to month 24 , and were not included in the present analysis

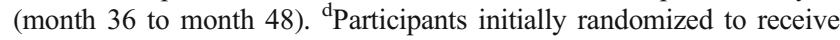
teriparatide (gray box) completed the study at month 12 and were not included in the present analysis (month 36 to month 48 ). ${ }^{\circ}$ Cumulative number of participants who discontinued the study during the first 24 months. ${ }^{f}$ Number of participants who discontinued the study between month 24 and month $36 .{ }^{\mathrm{g}}$ One subject was randomized to receive placebo in the initial treatment period but received romosozumab treatment. ${ }^{\mathrm{h}}$ Number of participants who discontinued the study between month 36 and month 48 . 'One participant had not undertaken the 48-month visit at the time of the analysis. PO, orally; QM, monthly; Q3M, every 3 months; Q6M, every 6 months; QW, weekly; SC, subcutaneous

their first course of romosozumab during the second-course period. Injection-site reactions, mostly mild in severity, were reported over the 12-month period in 10 (7.1\%) participants receiving a second course of romosozumab and in $2(7.4 \%)$ participants receiving their first course of romosozumab in the second-course period. Malignancy was reported in 5 (3.6\%) participants receiving a second course of romosozumab and in $1(3.7 \%)$ participant receiving her first course of romosozumab, and osteoarthritis was reported in $3(2.1 \%)$ participants and in $3(11.1 \%)$ participants, respectively. There were no reports of hyperostosis, hypocalcemia, 


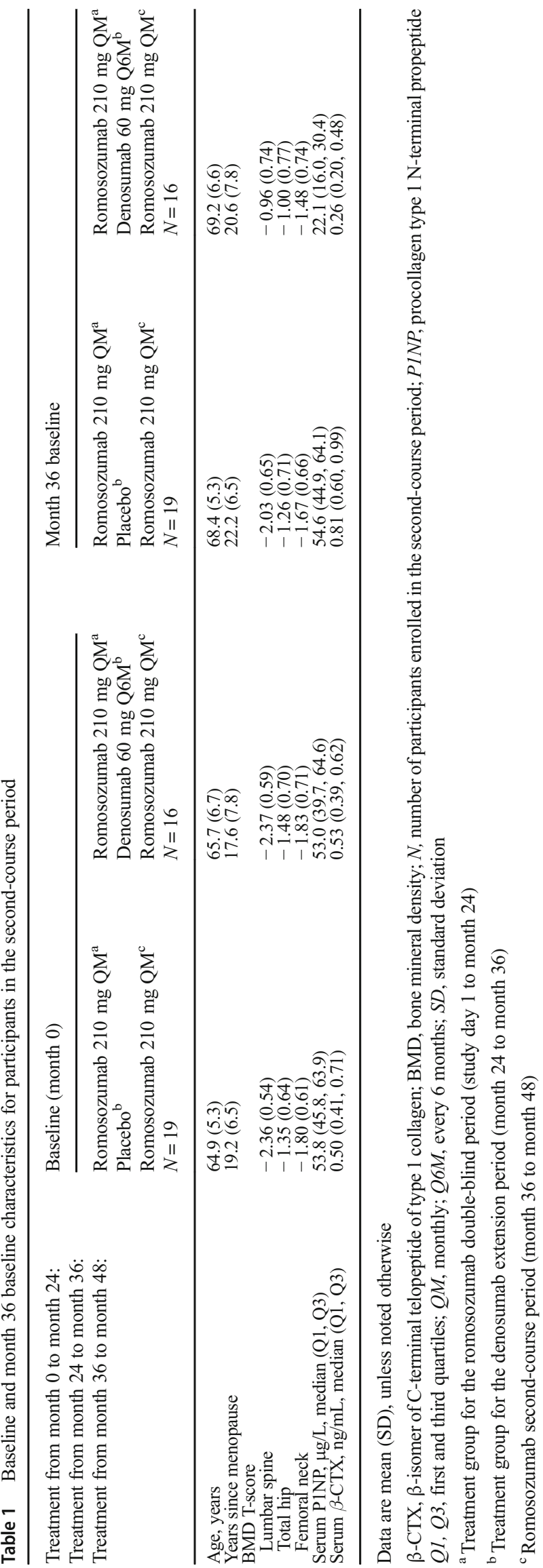

positively adjudicated osteonecrosis of the jaw, or positively adjudicated atypical femur fracture. Overall, subject incidence of adverse events in participants receiving a second course of romosozumab was similar to that in participants who received placebo between month 0 and month 12 (Table 3 ).

Of the 140 participants initially exposed to romosozumab, $33(23.6 \%)$ had at least one positive result for antiromosozumab antibody before receiving the first dose of romosozumab in the second-course period, with 6 and 8 participants having a positive result for antiromosozumab antibody at month 24 and month 36, respectively. Of these 33, 6 $(4.3 \%)$ had at least one positive result for neutralizing antiromosozumab antibody before receiving the first dose of romosozumab in the second-course period. During the second-course period, 11 of these 33 participants who had tested positive in the initial double-blind period tested positive for binding antibodies also during the denosumab extension period while the other 22 tested negative; only 1 of the 11 participants had antiromosozumab antibodies with neutralizing activity in vitro at the end of the second-course period. This 1 participant had tested positive for neutralizing antiromosozumab antibodies before receiving the first dose of romosozumab in the second-course period. No participant who previously tested negative to neutralizing antibodies developed neutralizing antiromosozumab antibodies during the second-course period. Two (1.4\%) participants previously antibody negative developed binding antiromosozumab antibodies during the second-course period, none with neutralizing activity. For the overall population, including the 1 participant who had antiromosozumab antibodies with in vitro neutralizing activity at the end of the second-course period, binding antibody status did not have any impact on the mean percentage changes from month 0 at the lumbar spine, total hip, and femoral neck BMD at both month 24 and month 48. During the second-course period, the frequency of overall adverse events by antibody status was comparable between antibody-positive and antibody-negative participants.

\section{Discussion}

The management of osteoporosis requires long-term therapy, which may include treatment with different agents, in sequence, over a patient's lifetime [12]. This study is the first to describe the efficacy and safety of a second course of romosozumab and provides further insight into potential treatment sequencing with this therapeutic agent.

In participants who received a second course of romosozumab following placebo, large BMD gains resulted at the lumbar spine, total hip, and femoral neck. These increases were similar in magnitude and rapidity to those observed during the initial 12 months of romosozumab treatment. The changes in bone turnover markers during the 
second course of romosozumab following placebo were also similar to those seen in the first romosozumab treatment course. The consistency and magnitude of the results of the second course of romosozumab compared with the first course of romosozumab therapy after 1 year without active therapy indicates that the responsiveness of the skeleton to sclerostin inhibition had fully reset within that time period, including the positive effect of romosozumab administration on modeling bone formation.

As bone mass and structure are major determinants of bone strength and thus fracture risk, it is reasonable to expect treatments that result in rapid and large gains in BMD to also result in faster and greater fracture risk reductions. Preclinical studies support maintenance of the relationship between bone mass and strength during romosozumab administration [13, 14] and our study showed that a second course of romosozumab following placebo produced large and rapid gains in BMD, which were similar to those observed during the first romosozumab administration. In randomized controlled studies, large and rapid BMD gains with initial romosozumab treatment were demonstrated to result in significant reductions in fracture risk in postmenopausal women with osteoporosis, in comparison with not only placebo in the pivotal FRActure study in postmenopausal woMen with ostEoporosis (FRAME) [4] but also with alendronate in the Active-controlled fRaCture study in postmenopausal women with osteoporosis at High risk (ARCH) [5]. In FRAME, the large BMD increases with an initial 1 year of romosozumab treatment were associated with a rapid reduction in fracture risk. This benefit extended during year 2 [4] and year 3 [15], when all participants received denosumab. During the 24month period after 12 months of romosozumab or placebo treatment, with all participants on denosumab in the second year, fewer fractures occurred in participants who first received romosozumab versus placebo in year 1 . This suggests a persistent benefit from rapid bone mass accrual, conveying a stronger foundation on which to transition to denosumab treatment.

In our study, we also assessed switching from denosumab to romosozumab. BMD was either further increased (lumbar spine) or maintained (total hip) with romosozumab following denosumab. This stands in contrast to the declines in BMD after denosumab treatment that have been documented in studies evaluating the switching from denosumab to teriparatide, an agent that stimulates bone formation but also increases bone resorption. In the DATA-Switch study [16], switching from denosumab to teriparatide showed transient losses in BMD at the spine and more pronounced losses at the hip in postmenopausal women with osteoporosis, demonstrating that the net effect of increased resorption from discontinuing denosumab cannot be fully compensated by teriparatide's effect of stimulation of bone formation. This is not surprising given that teriparatide therapy also has a pro- remodeling effect, which may negatively affect BMD at cortical sites [3, 17]. In contrast, in our study, continued BMD increases were observed at the lumbar spine, although these increases were of smaller magnitude than those observed in treatment-naïve patients. Hip BMD did not increase during the second course of romosozumab treatment following denosumab, but the bone-forming effects were sufficient to maintain BMD following denosumab discontinuation. This is explained by romosozumab's stimulation of bone formation together with its antiresorptive effect, fully offsetting the expected loss of bone mass associated with the increased bone resorption known to occur following denosumab discontinuation. While histomorphometric confirmatory data are not available, the observed profile of the bone turnover markers is consistent with this interpretation. Overall, data from our study suggest that patients on denosumab could be transitioned to romosozumab. However, this sequence provides a lower increase in BMD compared to transitioning from no treatment to romosozumab; further, continuing on denosumab would be expected to offer similar BMD gains at the spine and greater BMD gains at the hip relative to romosozumab following denosumab [18]. Additionally, the results from this study illustrate the resetting of skeletal responsiveness to romosozumab after a year off therapy, with a second course of romosozumab matching the increases in bone formation and decreases in bone resorption, as well as the BMD gains observed with the first administration of romosozumab. Additional studies would be needed to determine whether such skeletal responsiveness can be achieved at an earlier time point.

Pharmacokinetic and pharmacodynamic studies [11] have demonstrated treatment efficacy with a 12-month course of treatment with romosozumab, wherein there were no further sustained increases in markers of bone formation when treatment was continued for a second year. Because osteoporosis is a chronic disease and the effects of romosozumab are reversible, transitioning to another therapy after 1 year of romosozumab treatment is required in order to maintain or augment the BMD gains and fracture risk reduction achieved. The phase 3 studies confirm the efficacy of antiresorptive therapy (denosumab or alendronate) following romosozumab in both maintaining BMD increases and reducing fracture risk [4, 5]. In FRAME [4, 15], after 12 months of romosozumab, participants who received 24 months of denosumab continued to exhibit a lower fracture risk and continued to demonstrate BMD increases at the lumbar spine and total hip versus participants who initially received placebo in the first 12 months. In this phase 2 study, decreases in BMD were observed on discontinuation of romosozumab, while switching to denosumab resulted in further gains in BMD [10]. In ARCH [5], after 12 months of romosozumab versus alendronate, switching to alendronate for up to 24 months maintained lower fracture risk and BMD gains at the lumbar spine, total hip, 


\section{a. Lumbar Spine}
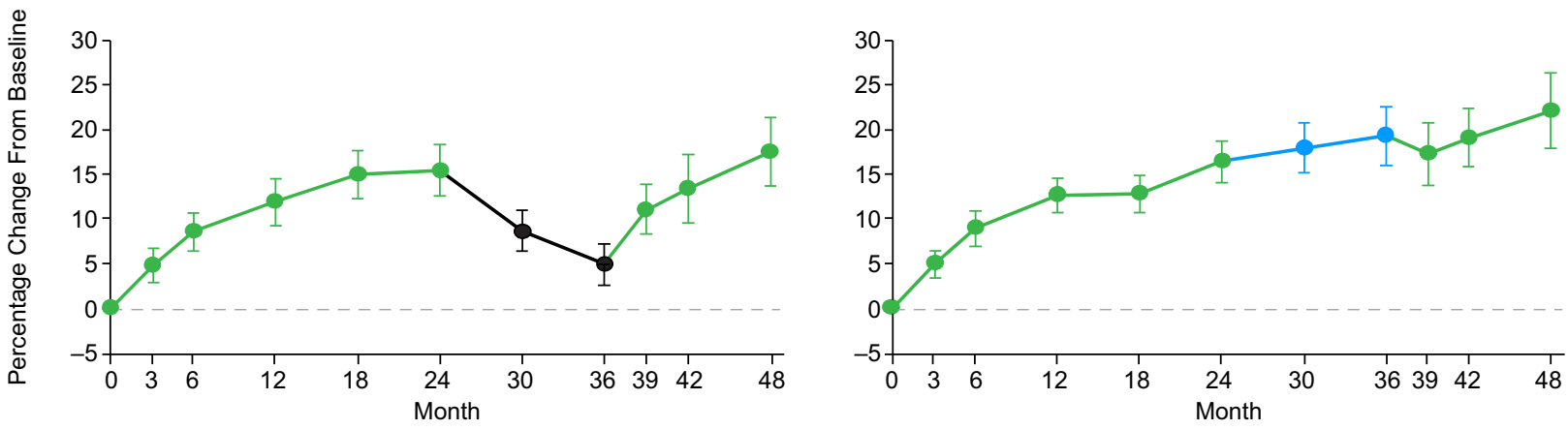

\section{b. Total Hip}
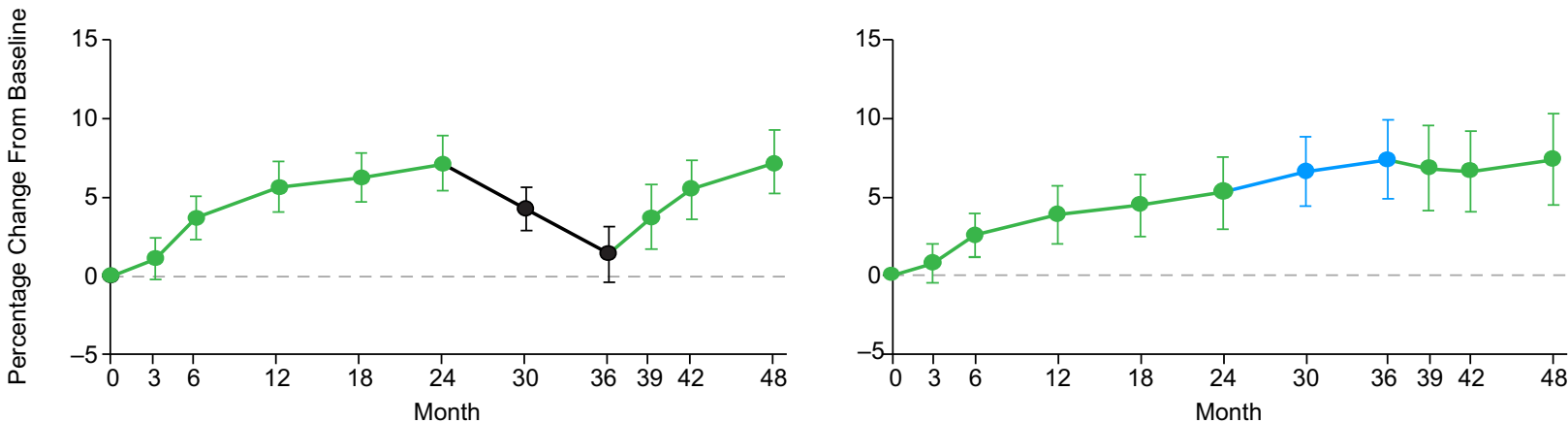

\section{c. P1NP}
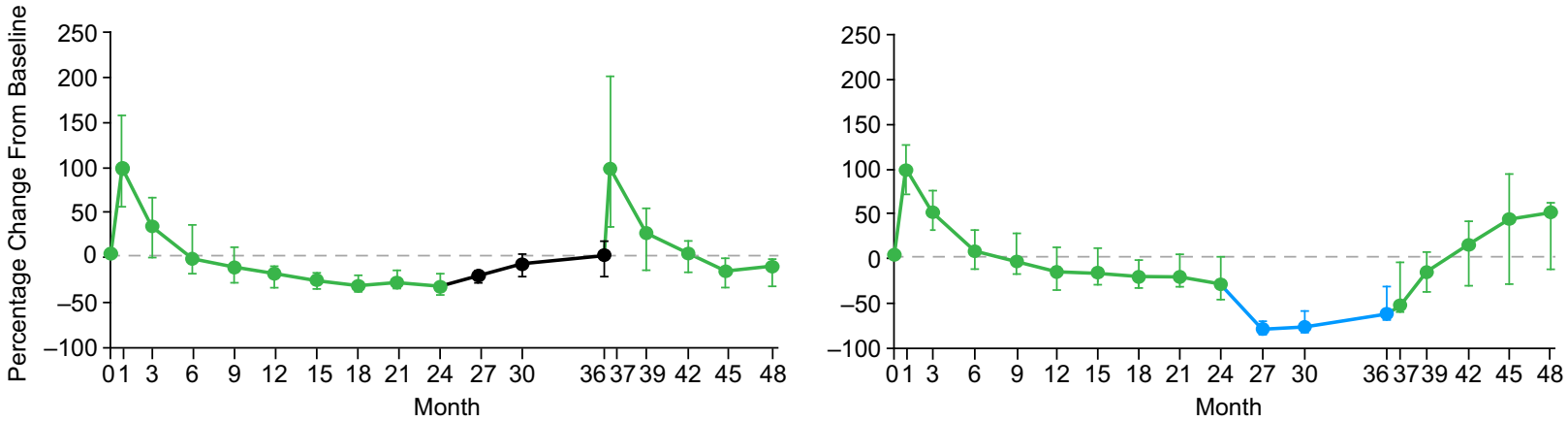

\section{d. $\beta$-CTX}

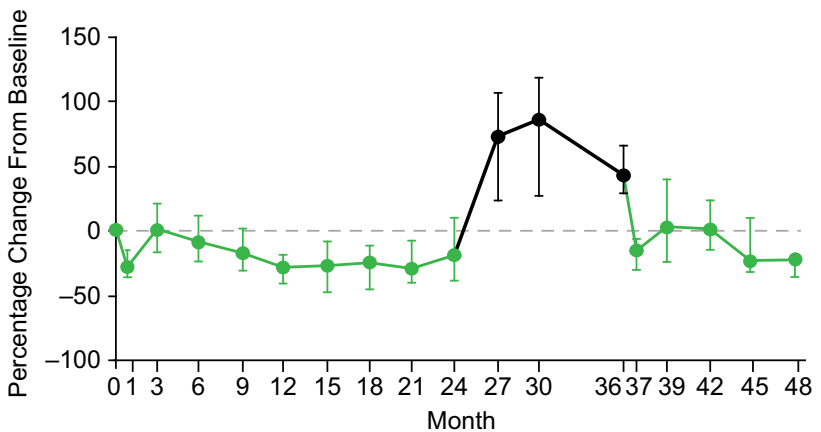

Fig. 3 Percentage change from month 0 to month 48 in lumbar spine BMD (a), total hip BMD (b), P1NP (c), and $\beta$-CTX (d). Data shown are for participants who had received romosozumab $210 \mathrm{mg}$ QM from month 0 to month $24(n=35)$ and had received either placebo $(n=19)$ or denosumab $60 \mathrm{mg}$ Q6M $(N=16)$ from month 24 to month 36, before receiving romosozumab $210 \mathrm{mg} \mathrm{QM}$ from month 36 to month 48 . Data

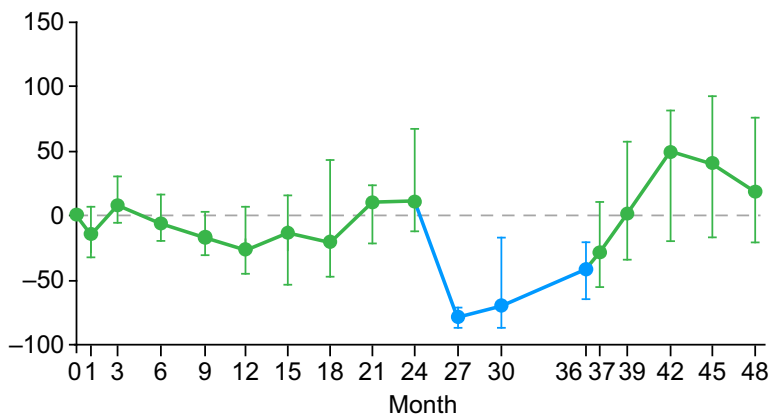

are mean $(95 \% \mathrm{CI})$ for $\mathrm{BMD}$ and median $(\mathrm{Q} 1, \mathrm{Q} 3)$ for P1NP and $\beta$-CTX $\beta$-CTX, $\beta$-isomer of C-terminal telopeptide of type 1 collagen; BMD, bone mineral density; $\mathrm{CI}$, confidence interval; P1NP, procollagen type 1 N-terminal propeptide; Q1, Q3, first and third quartiles; QM, monthly; Q6M, every 6 months 
and femoral neck compared with alendronate alone, supporting the long-term benefit of early treatment with romosozumab and sequencing romosozumab before an antiresorptive agent.

Studies have also compared switching from bisphosphonate therapy to either romosozumab or teriparatide. In the STudy evaluating effect of RomosozUmab Compared with Teriparatide in postmenopaUsal women with osteoporosis at high risk for fracture pReviously treated with bisphosphonatE therapy (STRUCTURE) [3], 12 months of romosozumab treatment resulted in BMD increases from month 0 at both the spine (9.8\%) and the hip (2.9\%), and significantly greater gains than with teriparatide (5.4\% and $-0.5 \%$, respectively). Larger increases in BMD were observed in participants switching from alendronate to romosozumab in the STRUCTURE study [3] compared with those switching from denosumab to romosozumab in the present study. This is likely the result of offsetting the rapid increases in bone resorption on discontinuing denosumab, which is not observed following discontinuation of bisphosphonates due to their skeletal incorporation.

A second course of romosozumab in this small group of participants did not show new safety findings, with an observed adverse event profile comparable to that of the first course of romosozumab treatment during month 0 to month 12 , and a low rate of neutralizing antibody development. As previously reported, no additional safety findings were observed with romosozumab treatment during month 12 to month 24 [10]. Overall, our data suggest that a second course of romosozumab was well tolerated in participants off osteoporosis treatment for a year or transitioning from denosumab. Consistent with other studies in women with postmenopausal osteoporosis comparing romosozumab with placebo (FRAME) [4] or teriparatide (STRUCTURE) [3], we did not observe an imbalance in cardiovascular adverse events with romosozumab administration in this study. Results from the ARCH study [5] indicated a higher incidence of positively adjudicated cardiovascular serious adverse events in postmenopausal women treated with romosozumab for 1 year $(2.5 \%)$ versus those treated with alendronate $(1.9 \%)$.

The main limitation of this study is the small sample size, adequate for the assessment of BMD but too small to evaluate fracture risk and low frequency safety signals. However, the BMD gains observed in participants receiving a second course of romosozumab after placebo were significant and similar to the gains observed in the initial romosozumab $210 \mathrm{mg}$ QM treatment group [8].

As would be expected with a reversible agent, in the absence of follow-on therapy, increases in BMD after initial romosozumab treatment reverse within a year off therapy; a second course of romosozumab again led to rapid and large BMD gains with similar rapidity and magnitude through its dual effect of increasing bone formation and decreasing bone resorption. The BMD effect of romosozumab after

Table 2 Percentage change in bone mineral density

Treatment from month 0 to month 24:

Treatment from month 24 to month 36 :

Treatment from month 36 to month 48 :

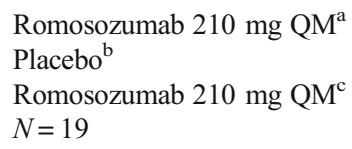

Romosozumab $210 \mathrm{mg} \mathrm{QM}^{\mathrm{a}}$ Denosumab $60 \mathrm{mg}$ Q6M ${ }^{\mathrm{b}}$ Romosozumab $210 \mathrm{mg} \mathrm{QM}$ $N=16$

\begin{tabular}{|c|c|}
\hline \multicolumn{2}{|l|}{ Lumbar spine } \\
\hline Month 0 to month 24 & $15.5(12.6,18.4)$ \\
\hline Month 24 to month 36 & $-9.1(-10.6,-7.5)$ \\
\hline Month 36 to month 48 & $12.4(10.4,14.5)$ \\
\hline Month 0 to month 48 & $17.6(13.7,21.4)$ \\
\hline \multicolumn{2}{|l|}{ Total hip } \\
\hline Month 0 to month 24 & $7.0(5.3,8.8)$ \\
\hline Month 24 to month 36 & $-5.3(-7.3,-3.3)$ \\
\hline Month 36 to month 48 & $6.0(4.5,7.5)$ \\
\hline Month 0 to month 48 & $7.1(5.2,9.1)$ \\
\hline \multicolumn{2}{|l|}{ Femoral neck } \\
\hline Month 0 to month 24 & $7.0(4.5,9.5)$ \\
\hline Month 24 to month 36 & $-4.3(-6.6,-1.9)$ \\
\hline Month 36 to month 48 & $6.3(3.3,9.4)$ \\
\hline Month 0 to month 48 & $8.6(5.4,11.8)$ \\
\hline \multicolumn{2}{|l|}{ Data are mean $\%(95 \% \mathrm{CI})$} \\
\hline \multicolumn{2}{|c|}{$C I$, confidence interval; $Q M$, monthly; $Q 6 M$, every 6 months } \\
\hline \multicolumn{2}{|c|}{${ }^{\mathrm{a}}$ Treatment group for the romosozumab double-blind period (study day 1 to month 24 ) } \\
\hline \multicolumn{2}{|c|}{${ }^{\mathrm{b}}$ Treatment group for the denosumab extension period (month 24 to month 36 ) } \\
\hline
\end{tabular}




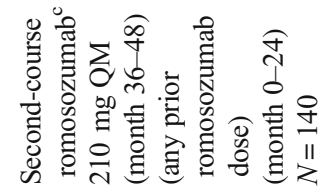

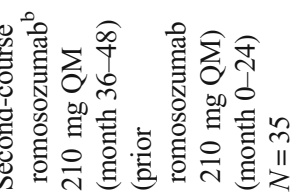

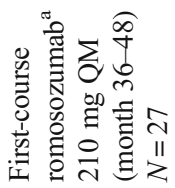

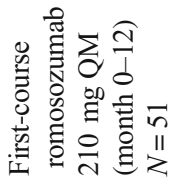

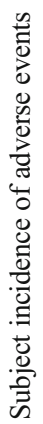

$\frac{m}{\circ}$

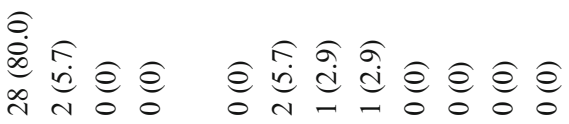

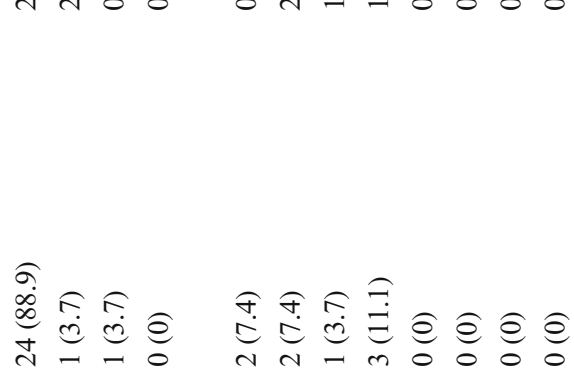

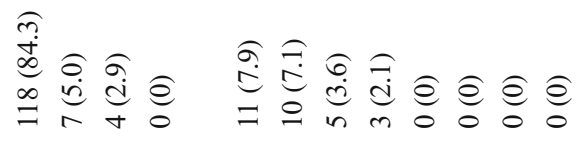

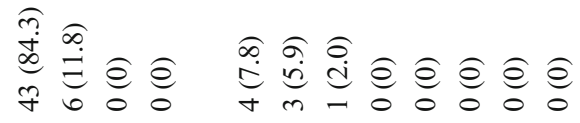

के$$
\text { 年 }
$$

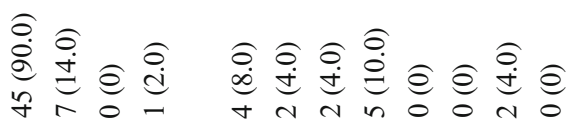
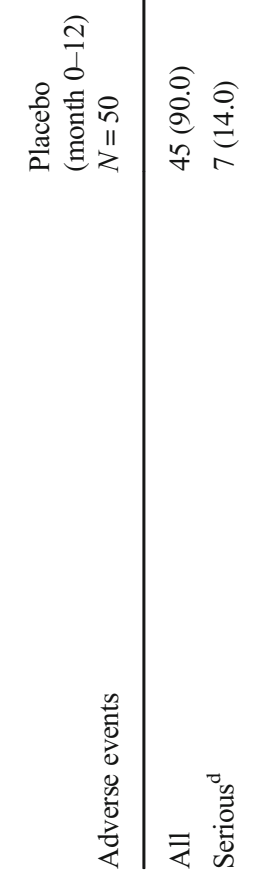
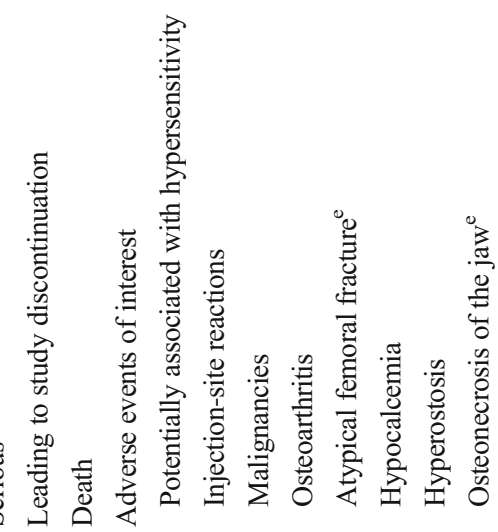

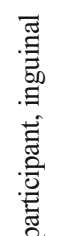

$\stackrel{\infty}{+}$

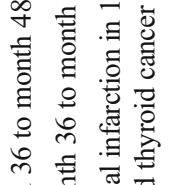

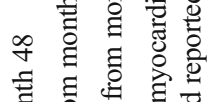

$\stackrel{8}{0}$

量

童

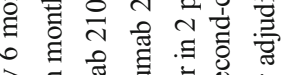

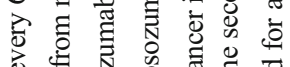

¿ $\sum_{0}$ O

ธ

语呈议

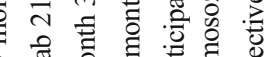

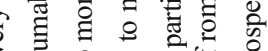

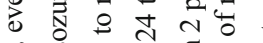

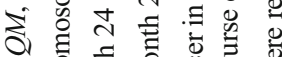

站言

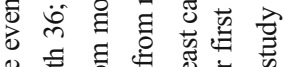

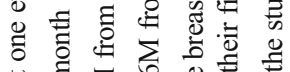

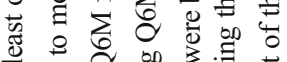

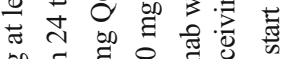

을

를 छ

을

$\sum$ च च

\% क्षे

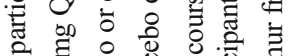

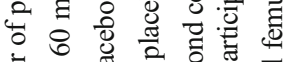

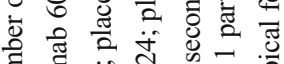

苟泀 on

เ चี

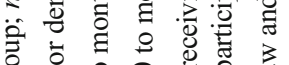

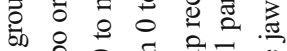

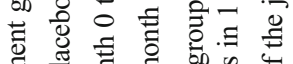

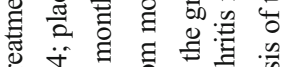

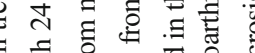

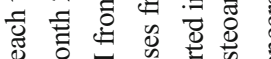

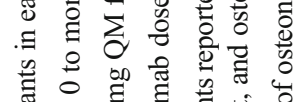

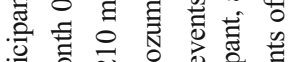

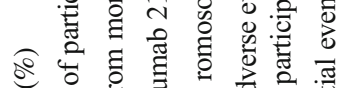

ङ

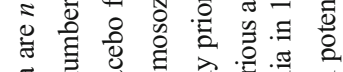

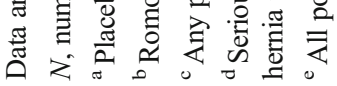


denosumab was less pronounced compared with the effect observed in treatment-naïve patients, but romosozumab compensated for the BMD declines associated with denosumab discontinuation, implying a significant bone-forming activity in these subjects as well. The data suggest that romosozumab is well tolerated and provide insight into osteoporosis treatment sequencing options for the long-term management of patients with osteoporosis. The effect of romosozumab on BMD is reversible upon discontinuation, and therefore should be followed by an antiresorptive agent to preserve BMD gains with the goal of maintaining fracture risk reduction over the long term. Since all currently available osteoporosis therapies are reversible over variable time frames, off-treatment patient response must be understood to properly sequence treatments to manage osteoporosis as a chronic disease. The effect of a second course of romosozumab provides needed data to inform possible long-term therapeutic decisions in those patients who remain at high risk for fracture.

Acknowledgments Amgen Inc., Astellas, and UCB Pharma sponsored this study (NCT00896532). All costs associated with the development of this manuscript were funded by Amgen Inc. and UCB Pharma. We thank the participants and investigators who were part of this study. Mandy Suggitt and Lisa Humphries (Amgen Inc.), and Martha Mutomba (on behalf of Amgen Inc.) provided medical writing support.

Funding Amgen Inc., Astellas, and UCB Pharma.

Data availability Qualified researchers may request data from Amgen clinical studies. Complete details are available at https://wwwext. amgen.com/science/clinical-trials/clinical-data-transparency-practices/.

\section{Compliance with ethical standards}

Conflicts of interest DLK has been an investigator for Amgen, Astellas, AstraZeneca, and Eli Lilly; a speaker and consultant for Amgen, Pfizer, and Eli Lilly; and a member of a drug safety advisory board for Merck. HGB has been an investigator for Amgen, Merck, and Shire; a consultant for Amgen, Merck, Radius Health, and Shire; a speaker for Amgen, Radius Health, and Shire; and a member of a data/safety monitoring board for Grünenthal. FM has no disclosures. EG has been a speaker for Amgen and Takeda, and an advisory board member for UCB and Alexion. SP has been an investigator for Pfizer, Amgen, Gedeon Richter, and Exeltis; a speaker for Bayer Schering, Novo Nordisk, Servier, Pfizer, MSD, Gedeon Richter, ProCare Health, Shionogi, and Teva; and an advisory board member for Novo Nordisk, Gedeon Richter, ProCare Health, and Shionogi. JM and RVD are employees of and hold stock in Amgen. CY was an employee of Amgen at the time of the study and may hold stock in Amgen, and is currently an employee of Cambridge Statistics Ltd. and holds stock in Cambridge Statistics Ltd. SY was an employee of Amgen at the time of the study and may hold stock in Amgen, and is currently an employee of Atara Biotherapeutics and holds stock in Atara Biotherapeutics. CL is an employee of and holds stock in UCB Pharma. AG was an employee of Amgen at the time of the study and may hold stock in Amgen, and is currently an employee of Corcept Therapeutics and holds stock options in Corcept Therapeutics.

Ethical approval All procedures performed in studies involving human participants were in accordance with the ethical standards of the institutional and/or national research committee and with the 1964 Helsinki Declaration and its later amendments or comparable ethical standards.

Informed consent Informed consent was obtained from all individual participants included in the study.

Open Access This article is distributed under the terms of the Creative Commons Attribution-NonCommercial 4.0 International License (http:// creativecommons.org/licenses/by-nc/4.0/), which permits any noncommercial use, distribution, and reproduction in any medium, provided you give appropriate credit to the original author(s) and the source, provide a link to the Creative Commons license, and indicate if changes were made.

\section{References}

1. Ferrari S, Adachi JD, Lippuner K, Zapalowski C, Miller PD, Reginster JY, Torring O, Kendler DL, Daizadeh NS, Wang A, O'Malley CD, Wagman RB, Libanati C, Lewiecki EM (2015) Further reductions in nonvertebral fracture rate with long-term denosumab treatment in the FREEDOM open-label extension and influence of hip bone mineral density after 3 years. Osteoporos Int 26:2763-2771

2. Cosman F, Crittenden DB, Ferrari S, Khan A, Lane NE, Lippuner K, Matsumoto T, Milmont CE, Libanati C, Grauer A (2018) FRAME study: the foundation effect of building bone with 1 year of romosozumab leads to continued lower fracture risk after transition to denosumab. J Bone Miner Res 33:1219-1226

3. Langdahl BL, Libanati C, Crittenden DB, Bolognese MA, Brown JP, Daizadeh NS, Dokoupilova E, Engelke K, Finkelstein JS, Genant HK, Goemaere S, Hyldstrup L, Jodar-Gimeno E, Keaveny TM, Kendler D, Lakatos P, Maddox J, Malouf J, Massari FE, Molina JF, Ulla MR, Grauer A (2017) Romosozumab (sclerostin monoclonal antibody) versus teriparatide in postmenopausal women with osteoporosis transitioning from oral bisphosphonate therapy: a randomised, open-label, phase 3 trial. Lancet 390:1585-1594

4. Cosman F, Crittenden DB, Adachi JD, Binkley N, Czerwinski E, Ferrari S, Hofbauer LC, Lau E, Lewiecki EM, Miyauchi A, Zerbini CA, Milmont CE, Chen L, Maddox J, Meisner PD, Libanati C, Grauer A (2016) Romosozumab treatment in postmenopausal women with osteoporosis. N Engl J Med 375:1532-1543

5. Saag KG, Petersen J, Brandi ML, Karaplis AC, Lorentzon M, Thomas T, Maddox J, Fan M, Meisner PD, Grauer A (2017) Romosozumab or alendronate for fracture prevention in women with osteoporosis. N Engl J Med 377:1417-1427

6. FORTEO $^{\circledR}$ (teriparatide [rDNA origin] injection) prescribing information, Eli Lilly and Company

7. TYMLOS ${ }^{\mathrm{TM}}$ (abaloparatide) prescribing information, Radius Health

8. McClung MR, Grauer A, Boonen S, Bolognese MA, Brown JP, Diez-Perez A, Langdahl BL, Reginster JY, Zanchetta JR, Wasserman SM, Katz L, Maddox J, Yang YC, Libanati C, Bone HG (2014) Romosozumab in postmenopausal women with low bone mineral density. N Engl J Med 370:412-420

9. Padhi D, Jang G, Stouch B, Fang L, Posvar E (2011) Single-dose, placebo-controlled, randomized study of AMG 785, a sclerostin monoclonal antibody. J Bone Miner Res 26:19-26

10. McClung MR, Brown JP, Diez-Perez A, Resch H, Caminis J, Meisner P, Bolognese MA, Goemaere S, Bone HG, Zanchetta JR, Maddox J, Bray S, Grauer A (2018) Effects of 24 months of treatment with romosozumab followed by 12 months of denosumab or 
placebo in postmenopausal women with low bone mineral density: a randomized, double-blind, phase 2, parallel group study. J Bone Miner Res 33:1397-1406

11. Padhi D, Allison M, Kivitz AJ, Gutierrez MJ, Stouch B, Wang C, Jang G (2014) Multiple doses of sclerostin antibody romosozumab in healthy men and postmenopausal women with low bone mass: a randomized, double-blind, placebo-controlled study. J Clin Pharmacol 54:168-178

12. Cosman F, Nieves JW, Dempster DW (2017) Treatment sequence matters: anabolic and antiresorptive therapy for osteoporosis. J Bone Miner Res 32:198-202

13. Ominsky MS, Boyd SK, Varela A, Jolette J, Felx M, Doyle N, Mellal N, Smith SY, Locher K, Buntich S, Pyrah I, Boyce RW (2017) Romosozumab improves bone mass and strength while maintaining bone quality in ovariectomized cynomolgus monkeys. J Bone Miner Res 32:788-801

14. Li X, Niu QT, Warmington KS, Asuncion FJ, Dwyer D, Grisanti M, Han CY, Stolina M, Eschenberg MJ, Kostenuik PJ, Simonet WS, Ominsky MS, Ke HZ (2014) Progressive increases in bone mass and bone strength in an ovariectomized rat model of osteoporosis after 26 weeks of treatment with a sclerostin antibody. Endocrinology 155:4785-4797

15. Lewiecki EM, Dinavahi RV, Lazaretti-Castro M, Ebeling PR, Adachi JD, Miyauchi A, Gielen E, Milmont CE, Libanati C,
Grauer A (2019) One year of romosozumab followed by two years of denosumab maintains fracture risk reductions: results of the FRAME extension study. J Bone Miner Res 34:419-428

16. Leder BZ, Tsai JN, Uihlein AV, Wallace PM, Lee H, Neer RM, Burnett-Bowie SA (2015) Denosumab and teriparatide transitions in postmenopausal osteoporosis (the DATA-Switch study): extension of a randomised controlled trial. Lancet 386:1147-1155

17. Macdonald HM, Nishiyama KK, Hanley DA, Boyd SK (2011) Changes in trabecular and cortical bone microarchitecture at peripheral sites associated with 18 months of teriparatide therapy in postmenopausal women with osteoporosis. Osteoporos Int 22:357-362

18. Bone HG, Wagman RB, Brandi ML, Brown JP, Chapurlat R, Cummings SR, Czerwinski E, Fahrleitner-Pammer A, Kendler DL, Lippuner K, Reginster JY, Roux C, Malouf J, Bradley MN, Daizadeh NS, Wang A, Dakin P, Pannacciulli N, Dempster DW, Papapoulos S (2017) 10 years of denosumab treatment in postmenopausal women with osteoporosis: results from the phase 3 randomised FREEDOM trial and open-label extension. Lancet Diabetes Endocrinol 5:513-523

Publisher's note Springer Nature remains neutral with regard to jurisdictional claims in published maps and institutional affiliations. 